\title{
GREEN AND CHAROPHYTE ALGAE IN BIOINDICATION OF WATER QUALITY OF THE SHAH ALAM RIVER (DISTRICT PESHAWAR, PAKISTAN)
}

Izaz KHURAM *, Zahir MUHAMMAD *, Nadeem AHMAD *, Rehman ULLAH * and Sophia BARINOVA **

* University of Peshawar, Khyber Pakhtunkhwa, Old Jamrud Road, Pakistan, PK-25120, izazkhuram@gmail.com,zahirbotany@gmail.com,nadeemgul77@gmail.com,rehmanbotany@gmail.com ** University of Haifa, Institute of Evolution, Mount Carmel, Abba Khoushi Avenue 199, Haifa, Israel, IL-3498838, Israel, sophia@evo.haifa.ac.il

DOI: 10.2478/trser-2019-0001

KEYWORDS: green algae, charophytes, bioindication, Shah Alam River.

\section{ABSTRACT}

58 species and infraspecies of Chlorophyta and Charophyta algae were observed in 60 samples collected in September 2017 from the Shah Alam River, Pakistan. The algal species richness and environmental variables increased down the river, except for $\mathrm{pH}$. Bioindication revealed low saline, low alkaline, middle streaming, and middle oxygenated water with low to middle organic pollution with Index saprobity S 1.48-1.73, Class 2-3 of Water Quality. The trophic state was eutrophic and meso-eutrophic with increasing eutrophication down the river. River Pollution Indices RPI demonstrated increasing of pollution in the Shah Alam River in comparison with the parallel part of the Kabul River.

ZUSAMMENFASSUNG: Süßwasser Grünalgen und Armleuchteralgen im Shah Alam Fluss (Distrikt Peshawar, Pakistan).

Zwecks Untersuchung der Grünalgen und Charophyta wurden im September 2017 aus dem Shah Alam River in Pakistan 60 Proben gesammelt, wobei insgesamt 58 Arten und infraspezifische Einheiten von Algen aus 60 Proben bestimmt wurden. Der Artenreichtum der Algen und die Werte der Umweltvariablen erhöhten sich - mit Ausnahme des pH-Werts - im unteren Abschnitt des Flusses. Mit Hilfe der Bioindikatoren lässt sich ein geringer Salzgehalt, schwach alkalines Wasser mittlerer Strömungsgeschwindigkeit und mittlerem Sauerstoffgehalt feststellen sowie eine niedrige bis mittlere organische Verschmutzung mit einem Saprobienindex von S 1.48-1.73 und Klasse 2-3 der Wasserqualität. Der trophische Zustand war eutroph und meso-eutroph mit steigenden Werten im unteren Abschnitt des Flusses. Der Wasserverschmutyungsindex RPI zeigte im Vergleich zum parallelen Teil des Kabul Flusses eine zunehmende Verschmutzung des Shah Alam River.

REZUMAT: Alge verzi și Charophyta din râul Shah Alam (Districtul Peshawar, Pakistan).

În vederea studiului algelor verzi şi a Charophytelor au fost prelevate din râul Shah Alam, Pakistan, 60 de probe, în septembrie 2017. Au fost identificate 58 de specii și infraspecii de alge Chlorophyta și Charophyta. Bogăția speciilor de alge și variabilele de mediu au crescut cu excepția pH-ului în sectorul din aval al râului. Bioindicatorii relevă un grad mic de salinizare și al alcalinității, o scurgere și oxigenare medie a apei prezentând o poluare organică scăzută până la medie și indicele de saprobitate S 1.48-1.73, clasa 2-3 a calității apei. Starea trofică a apei a fost eutrofă și mezo-eutrofă crescând în sectorul din aval al râului. Indicatorii de poluare a râului (RPI) au arătat o poluare crescândă în râul Shah Alam în comparație cu partea paralelă a râului Kabul. 


\section{INTRODUCTION}

Freshwater algae are very diverse organisms that occupied all natural environments where the water and light are present. Algae of aquatic habitats have a definite answer to changes in the water properties and, thus, are widely used as bioindicators of water quality and ecosystem status. (Rasiga et al., 1999; Barinova et al., 2006; Bellinger and Sigee, 2010)

It is crucial to assess the water quality in the rivers of Pakistan, which are major water sources for drinking, industry and agriculture in a semi-arid climate zone. The Pakistan rivers represent a part of the Indus River basin. Usually, they flow down from the mountains and later into flat relief valleys in which the settlements and industries are concentrated. The capital of Khyber Pakhtunkhwa is Peshawar, which is enriched with four main rivers: Kabul River, Nagoman River, Shah Alam River, and Bara River. Out of these four rivers, the Shah Alam River, is known for its anthropogenic load because diverse types of industry and agriculture are located on its banks, which directly dumps its waste into the river.

Freshwater algae are widely used in ecological assessment and monitoring of water quality (Stevenson, 2014; Barinova et al., 2006). It is very important to know about algal diversity in inland waters because most of algal species can be used as environmental indicators. Algal flora of the Peshawar District with special emphasis on Shah Alam River is under exploration.

A few scientific articles about its algae had been published (Sarim, 1989a, b, 1991; Sarim and Faridi, 1976; Khan and Faridi, 1977; Anjum and Faridi, 1985; Sarim and Ayaz, 2000; Sarim and Zaman, 2005; Anjum et al., 1980, 1982; Faridi et al., 1981; Sarim et al., 2008, 2011; Hussain et al., 1985, 2009, 2010, 2011, 2012; Khuram et al., 2014). This region of Pakistan is very loaded anthropogenically because it has flat territory in the Kabul River basin between high mountains with high capacity for agricultural production. The territory between the Warsak areas in the upper part of the Peshawar Plain and before the Swat River tributary input represents a region in which the Kabul River has many meanders in its flow with a minimum of three different flows in parallel. This territory is placed in the semi-arid climatic zone. Many birds are concentrated in this area near the riverbanks. Therefore, it is important to assess the water quality in one of these rivers - the Shah Alam River, the most southern one.

The aim of our present work was to reveal an algal species list with species-specific ecological preferences and abundance of algae from the Shah Alam River to assess the water quality dynamics based on bioindication methods.

\section{MATERIAL AND METHODS}

The Shah Alam River is almost 82 km long (Fig. 1), originates from Kabul River up to Machni and falls back to Kabul River after Mian Gujar.

Three algae sampling sites, Machni (1), Khazana (2), and Mian Gujar (3), were selected. 60 algal samples, 20 from each site, were collected during autumn (September 2017.

The collected algal samples ware fixed with $4 \%$ formalin (Edler and Elbrächter, 2010).

Taxonomic assessment of the algae specimens was carried out in the Department of Botany of University of Peshawar. For identification, the specimens were mounted on the glass slides and examined under a compound microscope. The specimens were identified with standard identification manuals (Collins, 1909; Transeau, 1951; Prescott, 1962; Wehr, 2002; Tiffany and Britton, 1952; Bellinger and Sigee, 2015) and updated with algaebase.org.

Temperature, $\mathrm{pH}$, electrical conductivity, total dissolved solids, salinity, and dissolved oxygen were measured on the sampling site by using a multiparameter water quality meter (HANNA HI 98194).

Species frequency was assessed with a six-score scale (Barinova et al., 2006). 
Species ecology data was derived from a database (Barinova et al., 2006) for seven ecological bioindication systems. A total list of revealed algal taxa was correlated with ecological database in the Office Access (Microsoft). Relationships of biological and environmental variables were calculated in Statistica 12.0 program. Diversity indices and comparative floristics were done in the GRAPHS program (Novakovsky, 2004). Algal abundance in each algological sample was calculated as a sum of scores of species in sixscores scale frequency (Barinova et al., 2006). Pearson coefficients were calculated with help of Wessa (2018). River Pollution Indices (RPI) were calculated as integral of each measured variable over the distance between sites (Barinova et al., 2006, 2010).

$$
R P I d=\sum_{i=1}^{n}(D i+D j) * 0.5 l / L
$$

Where $\mathrm{Di}, \mathrm{Dj}$ - an estimate of environmental variable or the corresponding index value for adjacent stations $\mathrm{i}$ and $\mathrm{j}$;

$\mathrm{l}$ - the distance between two adjacent stations $(\mathrm{km})$;

$\mathrm{L}$ - the total length of the river.

Index of saprobity S was calculated on the base of species ecology (Barinova et al., 2006) as:

$$
S=\sum_{i=1}^{n}\left(s_{i} \times h_{i}\right) / \sum_{i=1}^{n}\left(h_{i}\right)
$$

Where si is species-specific index saprobity, hi abundance scores of i-species, $\mathrm{S}$ is index saprobity of community on the station.

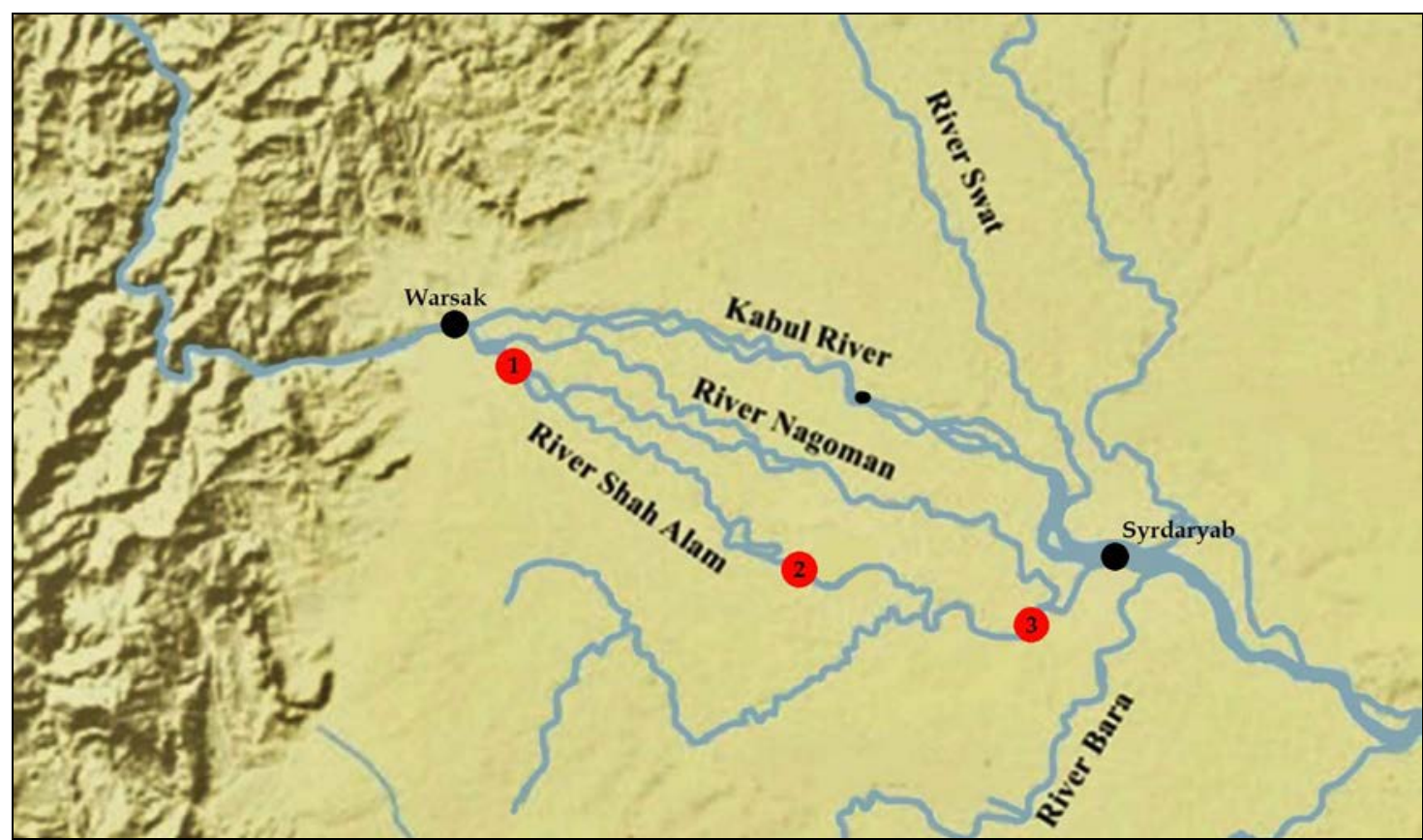

Figure 1: Sampling sites in the Shah Alam River: Machni-1, Khazana-2, and Mian Gujar-3. 


\section{RESULTS AND DISCUSSION}

Studied sites in the Shah Alam River (Fig. 1; Tab. 1) are placed in almost the same latitude and altitude about $300 \mathrm{~m}$ above sea level (a.s.l.). Total length of studied river part is $32.2 \mathrm{~km}$. The water temperature increased significantly from $18^{\circ} \mathrm{C}$ in Machni site to $22^{\circ} \mathrm{C}$ in Mian Gujar. In the same order other measured variables increased water conductivity, TDS, dissolved oxygen, and salinity as demonstrated in figure 2. The water $\mathrm{pH}$ in the river is changed in the opposite direction (Fig. 2). Therefore, the measured variable that fluctuated was salinity, and only $\mathrm{pH}$ was proto-opposite. This led us to assume that some processes in the river have a regular, not chaotic fluctuation.

Table 1: Physicochemical variables of water over sampling sites in Shah Alam River.

\begin{tabular}{|l|c|c|c|c|}
\hline Parameter & Unit & Machni & Khazana & Mian Gujar \\
\hline Number of site & & 1 & 2 & 3 \\
\hline Temperature & ${ }^{\circ} \mathrm{C}$ & 18.30 & 20.44 & 22.27 \\
\hline $\mathrm{pH}$ & & 9.10 & 8.48 & 8.01 \\
\hline Electrical conductivity & $\mu \mathrm{S} / \mathrm{cm}$ & 417 & 552 & 768 \\
\hline Total dissolved solids & $\mathrm{mg} / \mathrm{l}$ & 208 & 276 & 384 \\
\hline Salinity & $\mathrm{PSU}$ & 0.20 & 0.27 & 0.38 \\
\hline Dissolved oxygen & $\mathrm{mg} / \mathrm{l}$ & 34.1 & 47.4 & 85.2 \\
\hline Latitude & & 34.17096 & 34.09239 & 34.09021 \\
\hline Longitude & & 71.43341 & 71.61279 & 71.73102 \\
\hline Distance & $\mathrm{km}$ & 0 & 21.3 & 10.8 \\
\hline
\end{tabular}

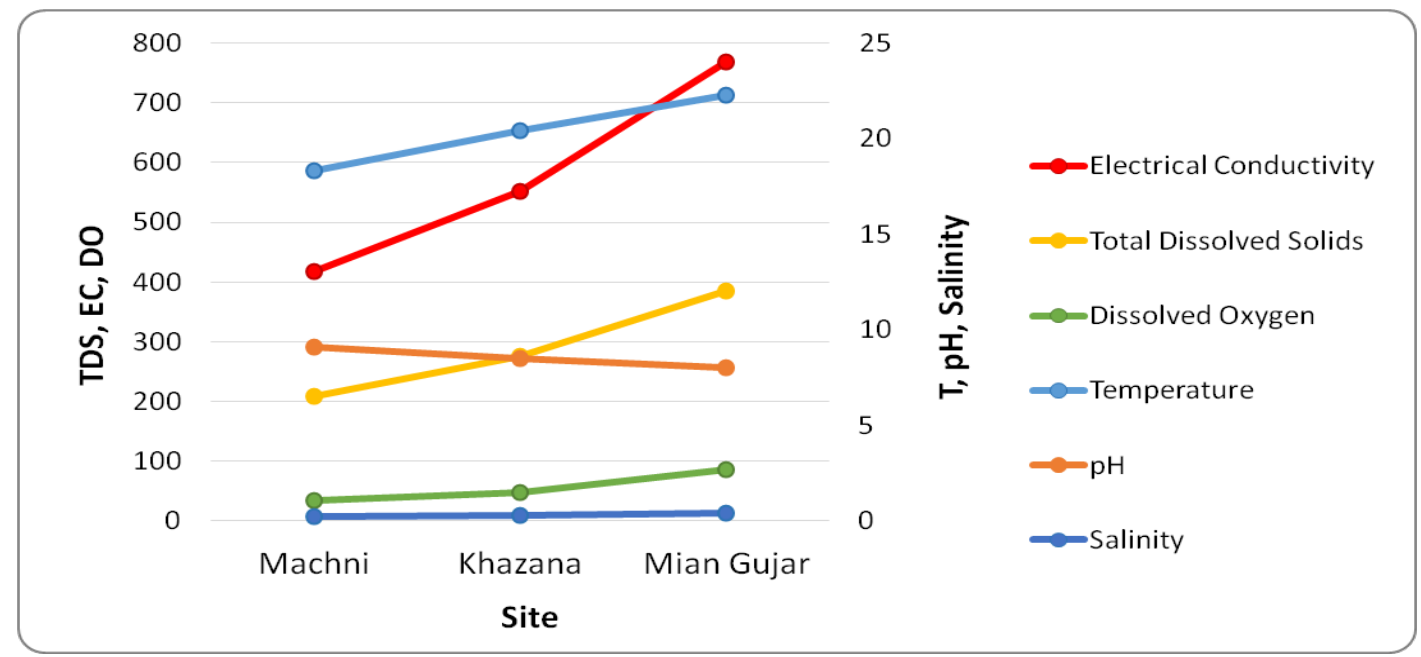

Figure 2: Dynamic of water $\mathrm{pH}$, salinity, algal species richness and index saprobity $\mathrm{S}$ over sampling sites in the Shah Alam River; the units as in the upper table 1.

16 genera with 58 species of unicellular and filamentous green and charophyte algae were identified from the collected samples of the Shah Alam River (Tab. 2). The genera are Chlamydomonas with three species, Chlorella - one sp., Chloroidium - one sp., Cladophora four sp., Closterium - five sp., Cosmarium - seven sp., Hyalotheca - one sp., Microspora two sp., Mougeotia - four sp., Mougeotiopsis - one sp., Oedogonium - five sp., Rhizoclonium - two sp., Pseudorhizoclonium - one sp., Schizomeris - one sp., Spirogyra - 12 sp., Temnogyra - one sp., Stigeoclonium - two sp., Uronema - one sp. and Zygnema - four sp. 
Table 2: Species diversity and autecology with a six-score scale frequency (Barinova et al., 2006) in sampling sites of the Shah Alam River; number of sites as in table 1.

\begin{tabular}{|c|c|c|c|c|c|c|c|c|c|c|}
\hline Taxa & 1 & 2 & 3 & Hab & Oxy & $\mathrm{pH}$ & Sal & Sap & $\mathrm{S}$ & Tro \\
\hline \multicolumn{11}{|l|}{ Chlorophyta } \\
\hline $\begin{array}{l}\text { Chlamydomonas angulosa } \\
\text { Dill O. }\end{array}$ & 0 & 2 & 3 & $\mathrm{P}$ & st & - & - & o-a & 1.80 & - \\
\hline $\begin{array}{l}\text { Chlamydomonas globosa } \\
\text { Snow J. W. }\end{array}$ & 0 & 3 & 0 & $\mathrm{P}, \mathrm{S}$ & - & - & - & o-a & 1.90 & - \\
\hline $\begin{array}{l}\text { Chlamydomonas sphagnicola } \\
\text { (Fritsch F. E.) Fritsch F. E. and Takeda H. }\end{array}$ & 2 & 0 & 2 & - & - & - & - & o & 1.20 & - \\
\hline $\begin{array}{l}\text { Chlorella vulgaris } \\
\text { Beyerinck }\end{array}$ & 0 & 0 & 3 & $\begin{array}{l}\mathrm{P}-\mathrm{B}, \\
\mathrm{pb}, \mathrm{S}\end{array}$ & - & - & hl & $\mathrm{a}$ & 3.10 & - \\
\hline $\begin{array}{l}\text { Chloroidium ellipsoideum (Gerneck) } \\
\text { Darienko, Gustavs, Mudimu, Menendez, } \\
\text { Schumann, Karsten, Friedl and Proschold }\end{array}$ & 0 & 2 & 2 & - & - & - & - & - & - & - \\
\hline $\begin{array}{l}\text { Cladophora fracta } \\
\text { (Müller and Vahl O. F.) Kützing }\end{array}$ & 5 & 5 & 4 & P-B & $\begin{array}{l}\text { st- } \\
\text { str }\end{array}$ & - & - & $\mathrm{b}$ & 2.30 & - \\
\hline $\begin{array}{l}\text { Cladophora glomerata var. glomerata } \\
\text { (Linnaeus) Kützing }\end{array}$ & 5 & 5 & 5 & P-B & $\begin{array}{l}\text { st- } \\
\text { str }\end{array}$ & alf & $\mathrm{i}$ & o-a & 1.90 & - \\
\hline $\begin{array}{l}\text { Cladophora glomerata var. crassior } \\
\text { (Agardh C.) Hoek }\end{array}$ & 5 & 3 & 0 & - & - & - & - & - & - & - \\
\hline $\begin{array}{l}\text { Cladophora rivularis } \\
\text { (Linnaeus) Hoek }\end{array}$ & 0 & 4 & 3 & - & - & - & - & - & - & - \\
\hline $\begin{array}{l}\text { Microspora amoena } \\
\text { (Kützing) Rabenhorst }\end{array}$ & 0 & 0 & 4 & B & - & - & - & $x-b$ & 0.80 & - \\
\hline $\begin{array}{l}\text { Oedogonium autumnale } \\
\text { Wittrock ex Hirn }\end{array}$ & 0 & 0 & 2 & - & - & - & - & - & - & - \\
\hline $\begin{array}{l}\text { Oedogonium crassum } \\
\text { Wittrock ex Hirn }\end{array}$ & 2 & 3 & 1 & - & - & - & - & - & - & - \\
\hline $\begin{array}{l}\text { Oedogonium intermedium } \\
\text { Wittrock ex Hirn }\end{array}$ & 0 & 3 & 0 & - & - & - & - & - & - & - \\
\hline $\begin{array}{l}\text { Oedogonium minus } \\
\text { Wittrock ex Hirn }\end{array}$ & 0 & 4 & 2 & - & - & - & - & - & - & - \\
\hline $\begin{array}{l}\text { Oedogonium multisporum } \\
\text { Wood H. C. ex Hirn }\end{array}$ & 2 & 0 & 0 & - & - & - & - & - & - & - \\
\hline $\begin{array}{l}\text { Pseudorhizoclonium africanum } \\
\text { (Kützing) Boedeker }\end{array}$ & 1 & 0 & 3 & - & - & - & - & - & - & - \\
\hline $\begin{array}{l}\text { Rhizoclonium crassipellitum } \\
\text { West and West G. S. }\end{array}$ & 1 & 0 & 3 & - & - & - & - & - & - & - \\
\hline $\begin{array}{l}\text { Rhizoclonium fontanum } \\
\text { Kützing }\end{array}$ & 0 & 3 & 2 & - & - & - & - & - & - & - \\
\hline $\begin{array}{l}\text { Schizomeris leibleinii } \\
\text { Kützing }\end{array}$ & 0 & 2 & 0 & - & - & - & - & b-a & 2.4 & - \\
\hline $\begin{array}{l}\text { Stigeoclonium fasciculare var. } \\
\text { glomeratum (Hazen) Islam A. K. }\end{array}$ & 2 & 4 & 5 & - & - & - & - & - & - & - \\
\hline $\begin{array}{l}\text { Stigeoclonium lubricum } \\
\text { (Dillwyn) Kützing }\end{array}$ & 2 & 3 & 3 & - & - & - & - & b-a & 2.5 & - \\
\hline $\begin{array}{l}\text { Uronema elongatum } \\
\text { Hodgetts }\end{array}$ & 1 & 0 & 0 & - & - & - & - & - & - & - \\
\hline
\end{tabular}


Table 2 (continued): Species diversity and autecology with a six-score scale frequency (Barinova et al., 2006) in sampling sites of the Shah Alam River; number of sites as in table 1.

\begin{tabular}{|c|c|c|c|c|c|c|c|c|c|c|}
\hline Taxa & 1 & 2 & 3 & Hab & Oxy & $\mathrm{pH}$ & Sal & Sap & S & Tro \\
\hline \multicolumn{11}{|l|}{ Charophyta } \\
\hline $\begin{array}{l}\text { Closterium acerosum } \\
\text { Ehrenberg ex Ralfs }\end{array}$ & 0 & 0 & 2 & P-B & $\begin{array}{l}\text { st- } \\
\text { str }\end{array}$ & ind & i & $a-o$ & 2.60 & e \\
\hline $\begin{array}{l}\text { Closterium lanceolatum } \\
\text { Kützing ex Ralfs }\end{array}$ & 1 & 4 & 2 & B & st & ind & - & - & - & e \\
\hline $\begin{array}{l}\text { Closterium leibleinii } \\
\text { Kützing ex Ralfs }\end{array}$ & 0 & 2 & 2 & P-B & $\begin{array}{l}\text { st- } \\
\text { str }\end{array}$ & ind & - & $a-0$ & 2.60 & e \\
\hline $\begin{array}{l}\text { Closterium littorale } \\
\text { Gay F. }\end{array}$ & 0 & 3 & 0 & P-B & - & ind & - & b-a & 2.40 & e \\
\hline $\begin{array}{l}\text { Closterium parvulum } \\
\text { Nägeli }\end{array}$ & 0 & 0 & 1 & P-B & - & ind & i & b & 2.00 & $\mathrm{~m}$ \\
\hline $\begin{array}{l}\text { Cosmarium botrytis } \\
\text { Meneghini ex Ralfs }\end{array}$ & 3 & 1 & 0 & P-B & $\begin{array}{l}\text { st- } \\
\text { str }\end{array}$ & ind & $\mathrm{i}$ & $0-\mathrm{a}$ & 1.90 & $\mathrm{~m}$ \\
\hline $\begin{array}{l}\text { Cosmarium granatum } \\
\text { Brébisson ex Ralfs }\end{array}$ & 0 & 5 & 0 & B & $\begin{array}{l}\text { st- } \\
\text { str }\end{array}$ & ind & i & o & 1.20 & $\mathrm{~m}$ \\
\hline $\begin{array}{l}\text { Cosmarium nitidulum } \\
\text { De Notaris }\end{array}$ & 2 & 0 & 0 & - & - & acf & - & - & - & $\mathrm{m}$ \\
\hline $\begin{array}{l}\text { Cosmarium quadrifarium } \\
\text { Lundell P. }\end{array}$ & 0 & 2 & 1 & - & - & acf & - & - & - & o \\
\hline $\begin{array}{l}\text { Cosmarium reniforme } \\
\text { (Ralfs) Archer W. }\end{array}$ & 0 & 0 & 1 & P-B & $\begin{array}{l}\text { st- } \\
\text { str }\end{array}$ & ind & hb & o & 1.00 & me \\
\hline $\begin{array}{l}\text { Cosmarium subcrenatum } \\
\text { Hantzsch }\end{array}$ & 5 & 1 & 3 & $\begin{array}{l}\text { B, } \\
\text { aer }\end{array}$ & aer & acf & - & o & 1.10 & $\mathrm{~m}$ \\
\hline $\begin{array}{l}\text { Cosmarium subimpressulum } \\
\text { Borge }\end{array}$ & 0 & 2 & 0 & - & - & acf & - & - & - & $\mathrm{m}$ \\
\hline Hyalotheca muсоsa Ralfs & 0 & 0 & 2 & P-B & - & acf & hb & - & - & o-m \\
\hline $\begin{array}{l}\text { Microspora stagnorum } \\
\text { (Kützing) Lagerheim }\end{array}$ & 0 & 2 & 3 & B & st & - & - & b-o & 1.60 & - \\
\hline $\begin{array}{l}\text { Mougeotia capucina } \\
\text { Agardh C. }\end{array}$ & 0 & 0 & 2 & B & - & - & - & o & 1.00 & - \\
\hline $\begin{array}{l}\text { Mougeotia genuflexa } \\
\text { (Roth) Agardh C. }\end{array}$ & 4 & 3 & 3 & B & - & - & - & $0-\mathrm{x}$ & 1.00 & - \\
\hline $\begin{array}{l}\text { Mougeotia punctata } \\
\text { (Wittrock) De Toni }\end{array}$ & 2 & 3 & 3 & B & - & - & - & o & 1.00 & - \\
\hline $\begin{array}{l}\text { Mougeotia robusta } \\
\text { (De Bary) Wittrock }\end{array}$ & 0 & 0 & 3 & B & - & - & - & 0 & 1.00 & - \\
\hline $\begin{array}{l}\text { Mougeotiopsis calospora } \\
\text { Palla }\end{array}$ & 3 & 0 & 5 & - & - & - & - & $x-b$ & 0.90 & - \\
\hline $\begin{array}{l}\text { Spirogyra communis } \\
\text { (Hassall) Kützing }\end{array}$ & 3 & 5 & 4 & B & st & - & - & $\mathrm{b}$ & 2.0 & - \\
\hline $\begin{array}{l}\text { Spirogyra crassa } \\
\text { (Kützing) Kützing }\end{array}$ & 3 & 2 & 0 & B & - & - & - & o-b & 1.5 & - \\
\hline $\begin{array}{l}\text { Spirogyra daedalea f. daedaloides } \\
\text { (Czurda) Poljansky V. }\end{array}$ & 5 & 4 & 4 & - & - & - & - & - & - & - \\
\hline $\begin{array}{l}\text { Spirogyra groenlandica } \\
\text { Rosenvinge }\end{array}$ & 4 & 0 & 3 & - & - & - & - & - & - & - \\
\hline $\begin{array}{l}\text { Spirogyra pratensis } \\
\text { Transeau }\end{array}$ & 3 & 0 & 4 & - & - & - & - & - & - & - \\
\hline $\begin{array}{l}\text { Spirogyra scrobiculata } \\
\text { (Stockmayer) Czurda }\end{array}$ & 2 & 5 & 2 & - & - & - & - & - & - & - \\
\hline
\end{tabular}


Table 2 (continued): Species diversity and autecology with a six-score scale frequency (Barinova et al., 2006) in sampling sites of the Shah Alam River; number of sites as in table 1.

\begin{tabular}{|c|c|c|c|c|c|c|c|c|c|c|}
\hline Таха & 1 & 2 & 3 & $\mathrm{Hab}$ & Oxy & $\mathrm{pH}$ & Sal & Sap & $S$ & Tro \\
\hline \multicolumn{11}{|l|}{ Charophyta } \\
\hline $\begin{array}{l}\text { Spirogyra setiformis } \\
\text { (Roth) Martens ex Meneghini }\end{array}$ & 0 & 1 & 4 & - & - & - & - & - & - & - \\
\hline $\begin{array}{l}\text { Spirogyra spreeiana } \\
\text { Rabenhorst }\end{array}$ & 5 & 0 & 0 & - & - & - & - & - & - & - \\
\hline $\begin{array}{l}\text { Spirogyra tenuissima } \\
\text { (Hassall) Kützing }\end{array}$ & 3 & 3 & 2 & B & - & - & - & o & 1.1 & - \\
\hline $\begin{array}{l}\text { Spirogyra tetrapla } \\
\text { Transeau }\end{array}$ & 0 & 2 & 1 & - & - & - & - & - & - & - \\
\hline $\begin{array}{l}\text { Spirogyra varians } \\
\text { (Hassall) Kützing }\end{array}$ & 0 & 4 & 3 & P-B & - & - & oh & b & 2.1 & - \\
\hline $\begin{array}{l}\text { Spirogyra weberi var. grevilleana } \\
\text { (Hassal) Kirchner O. }\end{array}$ & 0 & 2 & 3 & - & - & - & - & - & - & - \\
\hline $\begin{array}{l}\text { Temnogyra punctiformis } \\
\text { (Transeau) Yamagishi }\end{array}$ & 0 & 2 & 4 & - & - & - & - & - & - & - \\
\hline $\begin{array}{l}\text { Zygnema conspicuum } \\
\text { (Hassall) Transeau }\end{array}$ & 4 & 0 & 2 & B & - & - & - & o & 1.0 & - \\
\hline $\begin{array}{l}\text { Zygnema cyanosporum } \\
\text { Cleve }\end{array}$ & 0 & 3 & 0 & B & - & - & - & 0 & 1.0 & - \\
\hline $\begin{array}{l}\text { Zygnema pectinatum } \\
\text { (Vaucher) Agardh C. }\end{array}$ & 2 & 2 & 0 & B & $\begin{array}{l}\text { st- } \\
\text { str }\end{array}$ & - & oh & o & 1.0 & - \\
\hline $\begin{array}{l}\text { Zygnema sterile } \\
\text { Transeau }\end{array}$ & 0 & 1 & 5 & B & - & - & - & 0 & 1.0 & - \\
\hline Total number of indicators & 28 & 38 & 43 & 29 & 13 & 14 & 10 & 31 & 31 & 13 \\
\hline
\end{tabular}

Note - table 2: Substrate preferences. (Habitat): P - planktonic, P-B - plankto-benthic, B - benthic, Ep - epiphyte, S - soil. Temperature preferences (Temp): cool - cool-water, temp - temperate, eterm - eurythermic, warm - warm-water. Oxygenation and streaming (Reo): st - standing water, str - streaming water, st-str - low streaming water, ae - aerophiles. Halobity degree according Hustedt (1938-1939) (Hal): hb - oligohalobes-halophobes, i - oligohalobesindifferent, mh - mesohalobes, hl - halophiles. Acidity $(\mathrm{pH})$ degree according Hustedt (1957): alb-alkalibiontes, alf - alkaliphiles, ind - indifferent, acf - acidophiles, neu - neutrophiles as a part of indifferents. Species-specific Index of Saprobity (S). Self-purification zone preferences (Sap): $\mathrm{x}$ - xenosaprob, $\mathrm{x}-\mathrm{o}-\mathrm{xeno-oligosaprob,} \mathrm{o-x} \mathrm{-} \mathrm{oligo-xenosaprob,} \mathrm{x}-\mathrm{b}$ - xeno-beta-mesosaprob, o - oligosaprob, o-b - oligo-beta-msosaprob, b-o - beta-oligosaprob, o-a - oligo-alphamesosaprob, b - beta-mesosaprob, b-a - beta-alpha-mesosaprob, p-a - poly-alpha-mesosaprob, b-a - beta-alpha-mesosaprob, a - alpha-mesosaprob, a-o - alpha-oligosaprob. Saprobity degree according Watanabeet al. (1986) (D): sx - saproxen, es - eurosaprob, sp - saprophil. Nitrogen uptake metabolism (Aut-Het) (Van Dam et al., 1994): ats - nitrogen-autotrophic taxa, tolerating very small concentrations of organically bound nitrogen, ate - nitrogen-autotrophic taxa, tolerating elevated concentrations of organically bound nitrogen, hne - facultative nitrogen-heterotrophic taxa, needing periodically elevated concentrations of organically bound nitrogen, hce - obligatory nitrogen-heterotrophic taxa, needing continuously elevated concentrations of organically bound nitrogen. Trophic state (Tro) (Van Dam et al., 1994): ot - oligotraphentic, o-m - oligomesotraphentic, $\mathrm{m}$ - mesotraphentic, me - meso-eutraphentic, e - eutraphentic, o-e - oligo- to eutraphentic (hypereutraphentic). Class of water quality according EU scale. 
Table 2 reveals that species richness as well as total abundance scores are increasing down the river from Machni to Mian Gujar. Charophyta prevails in species number and abundance (as sum of scores) significantly in each studied site (Tab. 3) in the same direction. But the proportion of species and abundance in each site for green and charophyte algae was the same - Charophyta 60\%, and Chlorophyta $40 \%$ (Fig. 3).

Community structure changed down the river. In the upper Machni and Khazana sites the Charophyte species of Spirogyra and Cosmarium dominated. The algal community changed to domination of Mougeotiopsis and Zygnema also from Charophyta in lower Mian Gujar site. Green filamentous algae Cladophora were rich in all studied sites of the river.

Statistically calculated diversity indices were used for assessment of structural changes in algal communities over the studied sites in the Shah Alam River (Tabs. 4 and 5). Species richness was determined through Margalef index. Pielou's evenness index was used for species evenness. Species dominance was measured by using Simpson's index.
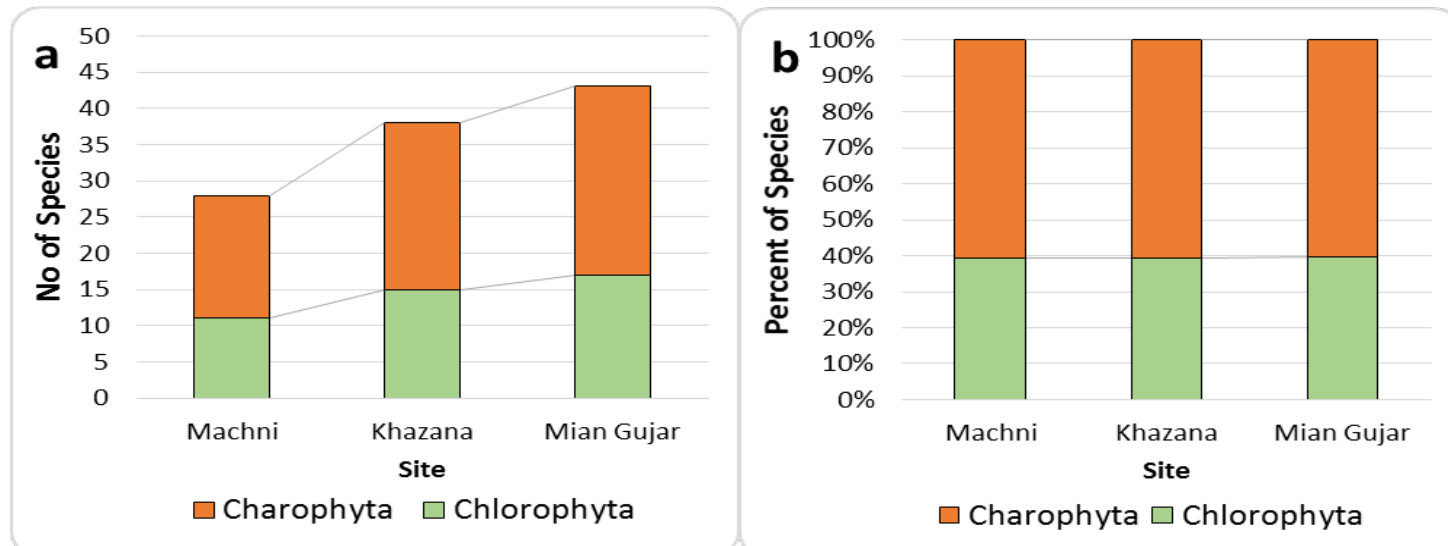

Figure 3: Distribution of Chlorophyta and Charophyta species richness over the sampling sites of the Shah Alam River. Species number (a), species percent (b).

Table 3: Distribution of species number, abundance, Chlorophyta and Charophyta species and genera over the Shah Alam River sampling sites.

\begin{tabular}{|l|c|c|c|c|c|}
\hline Division & Species & Genera & Machni & Khazana & Mian Gujar \\
\hline Chlorophyta & 23 & 9 & 11 & 15 & 17 \\
\hline Charophyta & 35 & 7 & 17 & 23 & 26 \\
\hline Total number of species & - & - & 28 & 38 & 43 \\
\hline Total abundance scores & - & - & 82 & 110 & 121 \\
\hline
\end{tabular}

Table 4: Statistics of algal diversity of Shah Alam River over sampling sites.

\begin{tabular}{|c|c|c|c|c|}
\hline \multirow{2}{*}{ No. } & \multirow{2}{*}{ Statistics } & \multicolumn{3}{|c|}{ Studied sites } \\
\cline { 3 - 5 } & & Machni & Khazana & Mian Gujar \\
\hline 1. & Total number of taxa & 58 & 58 & 58 \\
\hline 2. & Number of taxa as sp. richness & 28 & 38 & 43 \\
\hline 3. & Mean & 0.48 & 0.65 & 0.74 \\
\hline 4. & Standard error & 0.06 & 0.06 & 0.05 \\
\hline 5. & Variance & 0.25 & 0.22 & 0.19 \\
\hline 6. & Standard deviation & 0.50 & 0.47 & 0.44 \\
\hline
\end{tabular}


Table 5: Ecological diversity indices of algal communities of the Shah Alam River over sampling sites.

\begin{tabular}{|c|c|c|c|c|}
\hline \multirow{2}{*}{ No. } & \multirow{2}{*}{ Indices } & \multicolumn{3}{|c|}{ Studied sites } \\
\cline { 3 - 5 } & Margalef index & Machni & Khazana & Mian Gujar \\
\hline 1. & 8.10 & 10.17 & 11.17 \\
\hline 2. & Species Evenness & 0.95 & 0.94 & 0.94 \\
\hline 3. & Species Dominance & 0.03 & 0.02 & 0.02 \\
\hline 4. & $\begin{array}{c}\text { Combined Ecological Diversity } \\
\text { as Shannon index }\end{array}$ & 3.33 & 3.63 & 3.76 \\
\hline 5. & Index Saprobity S & 1.48 & 1.73 & 1.57 \\
\hline
\end{tabular}

We observed that community structure regularly changed in statistics variables. Species richness, mean, and Shannon index increased whereas species variance, standard deviation, evenness and dominance decreased down the river. Table 5 also included results of index saprobity $S$ calculation that show fluctuation over the studied sites from Class 2 of Water Quality in upper Machni site to Class 3 in sites Khazana and Mian Gujar. Therefore, we observed that organic pollution in the Shah Alam River was not so large and major sources of pollution can be found in the middle part of the river where Index saprobity $S=1.73$ was maxim. As illustrated in table 1 and figure 2, water variables changed regularly. We calculated Pearson coefficients for all measured environmental variables, algal species richness, and Index saprobity S. Only two coefficients show significant correlation. Water $\mathrm{pH}$ and salinity were strongly negatively correlated $(-0.98, \mathrm{p}<0.05)$, and also water $\mathrm{pH}$ and species richness $(-0.99$, $\mathrm{p}<0.03$ ) were correlated negatively.

We assume that water $\mathrm{pH}$ was influenced not only by environmental parameters in the river but also impacted species composition in algal community.

Our calculation show increasing organic pollution in the Shah Alam River in comparison of the parallel flow part of the Kabul River (Barinova et al., 2016) where saprobity varied between 1.55 and 1.59 reflect Class 3 of Water Quality.

We analysed bioindicator species representation in each studied site as well as distribution of specific groups over river communities and between them. Table 2 and figure 4 shows that benthic species strongly prevail in all studied sites, but number of plankto-benthic and planktonic inhabitants was rich in the lower sites. Increasing ecological group indicators of low oxygenated waters reflects the slowing of the river flow and the decrease of dissolved oxygen in the water down the river.

Sufficient decreasing in water $\mathrm{pH}$ is reflected in alkaliphilic species and increasing of indicators of low alkaline waters (Fig. 4) down the river. Salinity indicators were diverse in the lower Mian Gujar site only. This means low saline waters over the whole river. Indication of organic pollution dynamic in studied sites with help of saprobity groups (Tab. 2) that combined in the Water Quality classes show increasing of Class 3 and Class 4 indicators down the river (Fig. 4) that can be assessed as increase of organic pollution.

Assessment of trophic state dynamic over the river sampling sites show (Fig. 4) increasing of eutrophic and meso-eutrophic indicators from Machni to Mian Gujar. Therefore, in the Shah Alam River, not only organic pollution increase was detected but also trophic state down the river flow. 


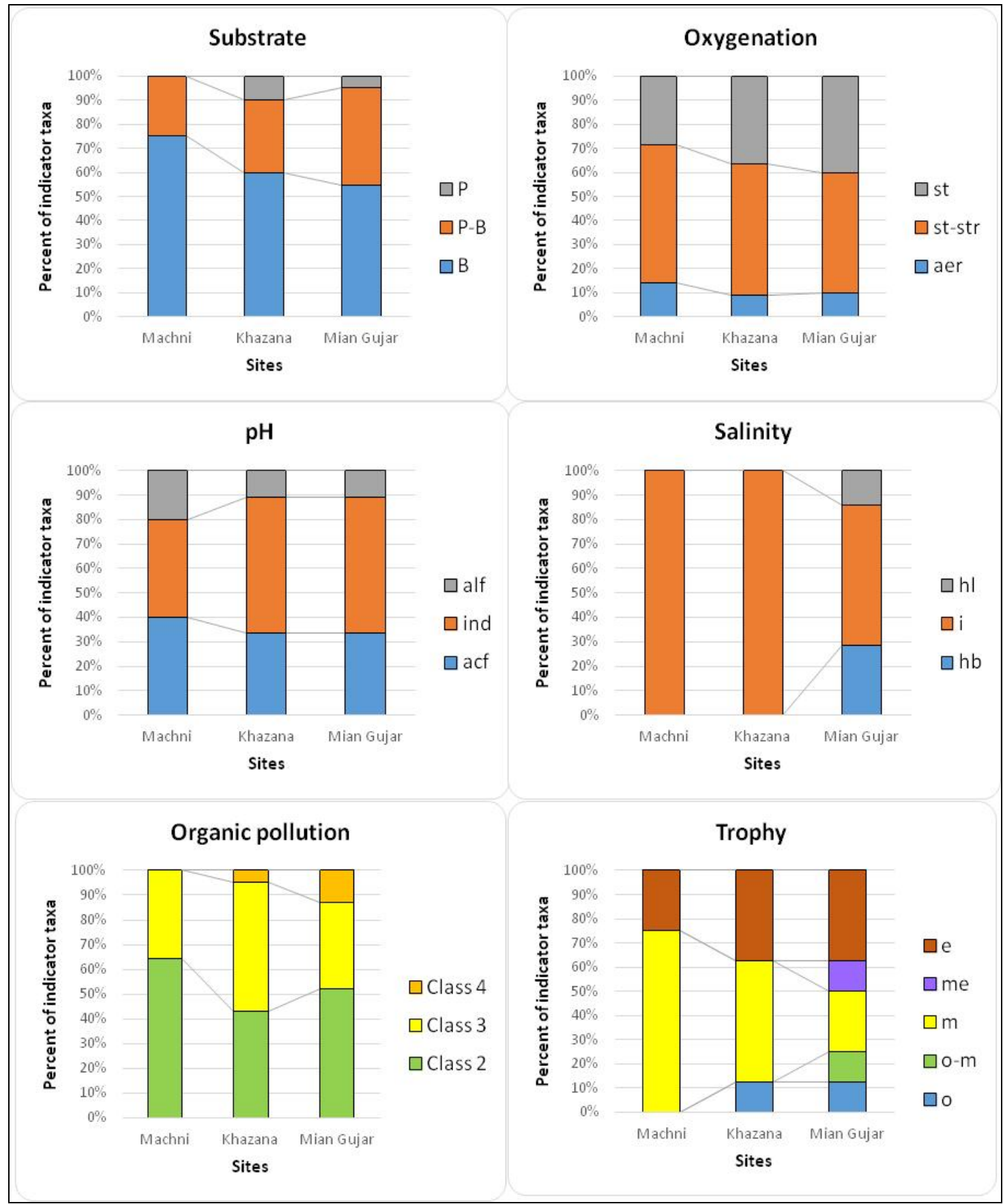

Figure 4: Distribution of indicator species in the ecological groups over the sampling sites of the Shah Alam River.

Classes of Water Quality in the Organic pollution plot are in EU colour code.

Abbreviations of ecological group as in table 2. 
We compared community structure in studied sites with help of comparative floristic program GRAPHS. The dendrite in figure 5 shows that Mian Gujar community is the richest including two other site communities to more than $50 \%$ of species. It reflects high capacity of revealed algal flora to develop even under pollution stress in the Shah Alam River and community in Mian Gujar that can be marked as floristic core of the river algal diversity.

Dendrogram of community similarity (Fig. 6) revealed more similarity between two lower sites community than uppermost Machni site. This can point to changing environment properties in the studied river after Machni site.

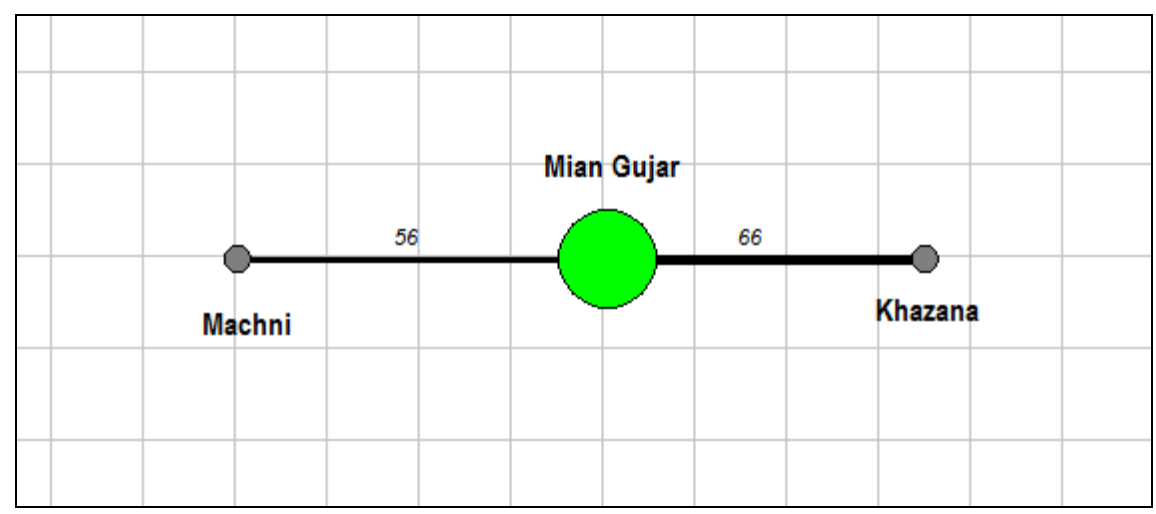

Figure 5: Dendrite of species richness overlapping in sampling sites communities of the Shah Alam River.

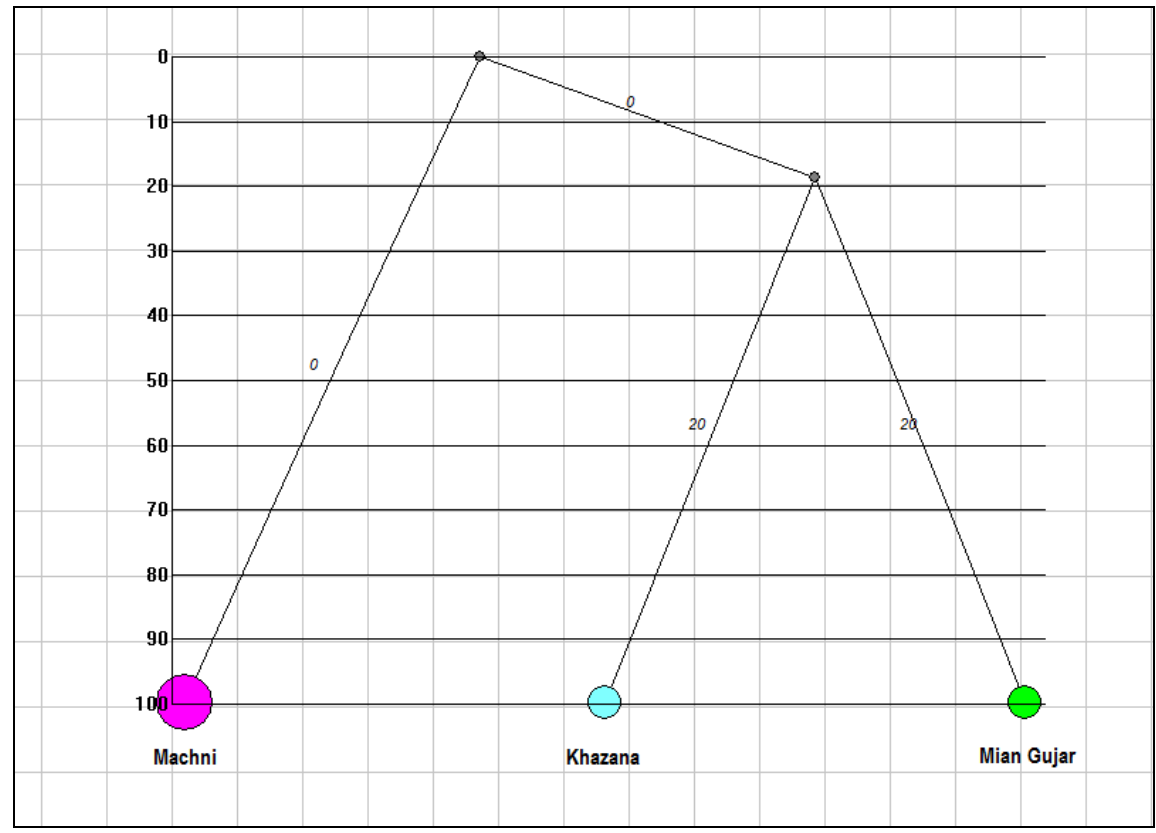

Figure 6: Dendrogram of species richness similarity for sampling sites communities of the Shah Alam River. 
Figure 7 presents surface plots that have been generated statistically for revealing relationships of algal diversity and environmental variables in the Shah Alam River. Plots in figures $7 \mathrm{a}$ and $7 \mathrm{~b}$ represent influences of water quality variables to species richness and show that the richest community can be developed in low TDS and low pH of water and preferred more saline water with low oxygenation. At the same time, the green algae that contain no more than $40 \%$ of species in each site survived better with the increasing water temperature and salinity, and decreasing of conductivity and dissolved oxygen (Figs. 7c and 7d). The charophytes, that prevail in community of each site, preferred lower TDS, $\mathrm{pH}$ and oxygen but increased with salinity increasing in the Shah Alam River (Figs. 7e and 7f).

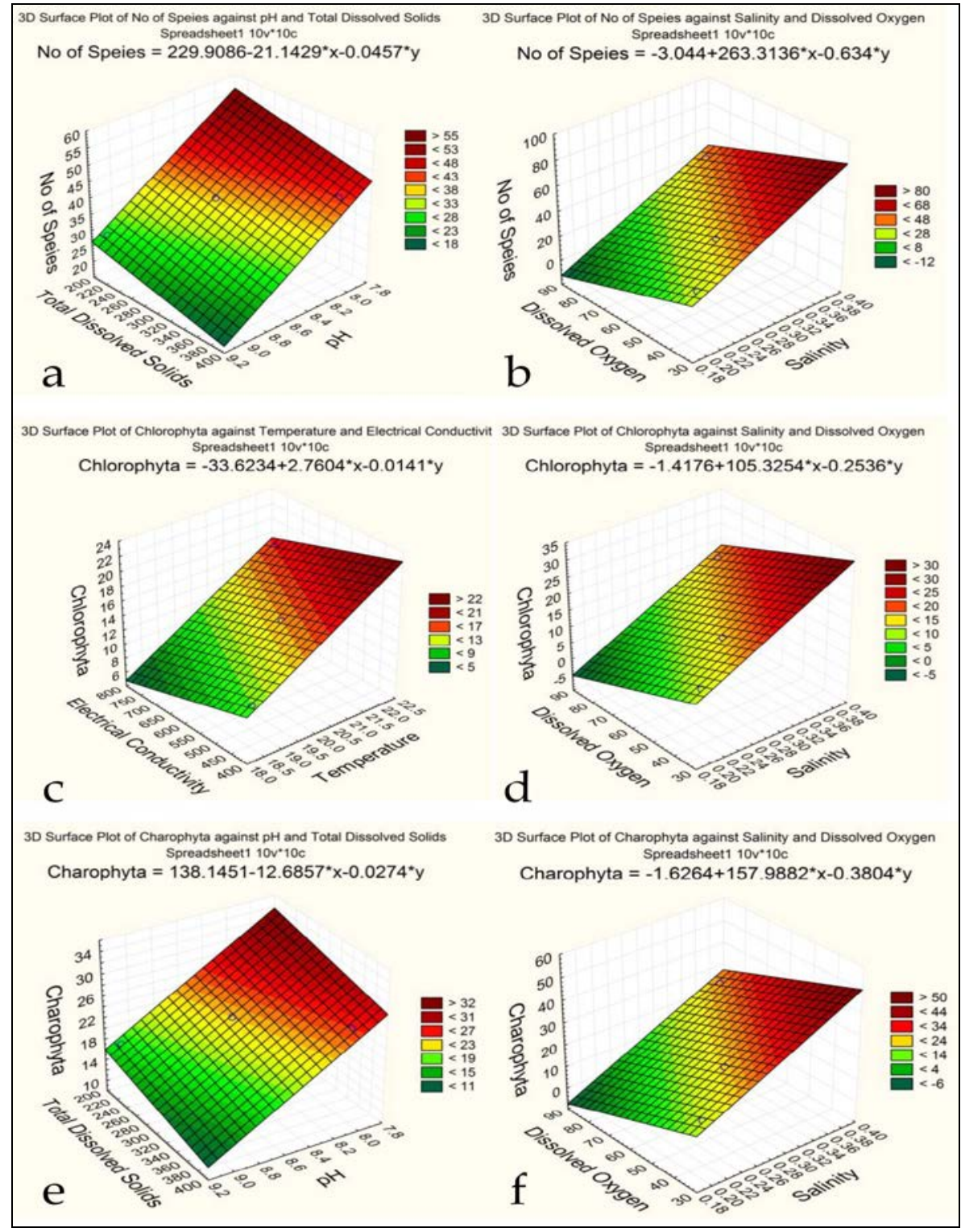

Figure 7: Statistical surface plots of species richness and environmental variables in the Shah Alam River. 
The integral indices RPI (River Pollution Index) were calculated for the Shah Alam River (Tab. 6) as has been described previously (Khuram et al., 2017). We observed that the integral RPI for the Shah Alam River is similar to variables in the middle site Khazana. RPI index has been calculated earlier for the Kabul River main stream (Khuram et al., 2017) that flow in parallel with the Shah Alam River from Warsak to Syrdaryab sites. We compared the data about Kabul and Shah Alam rivers and calculated RPI for parallel stream Shah Alam including upper site Warsak in the Kabul River and site Syrdaryab that placed below the Mian Gujar site in the Shah Alam River. Total length of the river between Warsak and Sardaryab is $51.5 \mathrm{~km}$. Total length of the river channel (across the studied sites of the Shah Alam River) between Warsak and Sardaryab is $51.5 \mathrm{~km}$. The distance between Warsak and Machni is 10.15 $\mathrm{km}$, while the distance from Machni to Khazana is $21.32 \mathrm{~km}$. The distance from Khazana to Mian Gujar is $10.83 \mathrm{~km}$, and between Mian Gujar and Sardaryab is 9.2. Second column in table 6 represents RPI calculation for the Shah Alam River including sites in the Kabul River. Comparisons show that environmental integral RPI and RPI for the Charophyta species richness were slightly lower in the Kabul River part including sites before and after the Shah Alam River than in studied river itself. Only total species richness RPI and RPI of Chlorophyta species number were greater in part with Kabul sites than in the Shah Alam River itself. Therefore, the calculation of RPI shows not only increasing water pollution in the Shah Alam River more than in the parallel Kabul River, but also a decreasing of total species richness, and a decrease in Chlorophyta in particular. RPI let us choose the Kazansa site as it reflects mean parameters of algal community and environment as reference for the whole Shah Alam River.

Table 6: River Pollution Indices (RPI) for variables of the Shah Alam River itself on the base of table 1 (first column) and with upper and lower stations of the parallel part of the Kabul River on the base of data according Khuram et al., 2017 (second column).

\begin{tabular}{|l|c|c|}
\hline \multicolumn{1}{|c|}{ Variable } & $\begin{array}{c}\text { RPI Machni-Khazana-Mian } \\
\text { Gujar }\end{array}$ & $\begin{array}{c}\text { RPI Warsak-Machni-Khazana- } \\
\text { Mian Gujar-Syrdaryab }\end{array}$ \\
\hline Temperature & 20.0 & 19.8 \\
\hline pH & 8.6 & 8.4 \\
\hline EC & 543.6 & 498.9 \\
\hline TDS & 271.6 & 247.3 \\
\hline Salinity & 0.3 & 0.29 \\
\hline Dissolved oxygen & 49.4 & 42.9 \\
\hline Number of species & 35.5 & 44.2 \\
\hline Chlorophyta species & 14.0 & 26.4 \\
\hline Charophyta species & 21.5 & 17.8 \\
\hline $\begin{array}{l}\text { Abundance, } \\
\text { sum of scores }\end{array}$ & 102.6 & - \\
\hline $\begin{array}{l}\text { Chlorophyta, } \\
\text { sum of scores }\end{array}$ & 40.2 & - \\
\hline $\begin{array}{l}\text { Charophyta, } \\
\text { sum of scores }\end{array}$ & 62.4 & - \\
\hline
\end{tabular}




\section{CONCLUSIONS}

The study of the diversity of algae in the Shah Alam River is still far from complete. Nevertheless, it revealed 58 algal taxa of species and subspecies levels which helped us characterize the changes in algal community under river pollution conditions.

We revealed that charophytes prevail in each of the three studied sites and contain $60 \%$ of community. Species richness, abundance as sum of scores, and community structure complexity increased down the river, whereas statistically calculated dominance decreased. Algal species-indicators of environment quality were revealed for seven ecological variables and demonstrated prevailing of benthic algae, but plankto-benthic and planktonic inhabitants enriched the community down the river. Diversity analysis showed that algal community in the Shah Alam River contained the green and charophytic algae. Community dominants changed from Cladophora-Spirogyra-Cosmarium at the upper site to Cladophora-MougeotiopsisZygnema species down the river.

Bioindication method revealed that algal community in the river prefer low saline, low alkaline, middle streaming, and middle oxygenated water with low to middle organic pollution. The trophic state of the river waters was defined as eutrophic and meso-eutrophic and demonstrated that in the Shah Alam River not only organic pollution increased but also trophic state down the river. Major water variables that regulated the total species richness were TDS, oxygen and $\mathrm{pH}$ as negative, and salinity (as a part of organic pollution) as positive for species richness increasing. Whereas salinity stimulated the development of both green and charophyte algae, while the water temperature was stimulating for greens. The richest community in the lower site of Mian Gujar can be chosen as the floristic core of algal flora of the Shah Alam River. The statistical comparison revealed that environmental impact to the river algal community starts after Machni site, in Khazana site with increasing water pollution which then self-purifies in the Mian Gujar site.

River Pollution Indices (RPI) demonstrated increasing pollution in the Shah Alam River in comparison with the parallel part of the Kabul River main stream and led us to choose the Khazana site as reference for whole Shah Alam River for future monitoring.

Therefore, these results can confirm the effectiveness of bioindication methods for water quality assessment in the Kabul River basin as well as its effectiveness for future use including the water monitoring in Pakistan.

\section{ACKNOWLEDGEMENTS}

This work has been partly supported by the Israeli Ministry of Absorption. 


\section{REFERENCES}

1. Anjum G. and Faridi M. A. F., 1985 - Algae in dry soil of N. W. F. P., Pakistan, Pakistan Journal of Botany, 17, 2, 257-261.

2. Anjum G., Hussain F. and Haq I., 1980 - Algae from turtle shells, Pakistan Journal of Botany, 12, 2, 201-203.

3. Anjum G., Jabeen T., Hussain F. and Faridi M. A. F., 1982 - Some soil-binding algae from Peshawar, Pakistan, Pakistan Journal of Botany, 14, 1, 107-109.

4. Barinova S. S., Medvedeva L. A. and Anissimova O. V., 2006 - Diversity of algal indicators in environmental assessment, Tel Aviv, Pilies Studio, 458. (in Russian)

5. Barinova S., Tavassi M., Glassman H. and Nevo E., 2010 - Algal indication of pollution in the Lower Jordan River, Israel, Applied Ecology and Environmental Research, 8, 1, 19-38.

6. Barinova S., Khuram I., Asadullah Ahmad N., Jan S. and Shin D. H., 2016 - How water quality in the Kabul River, Pakistan, can be determined with algal bio-indication, Advance Studies in Biology, 8, 4, 151-171.

7. Bellinger E. G. and Sigee D. C., 2010 - Fresh water algae (identification and use as bioindicators), John Wiley and Sons, Chippenham, Wilts, UK, 284.

8. Bellinger E. G. and Sigee D. C., 2015 - Freshwater algae: identification and use as bioindicators, John Wiley and Sons, 284.

9. Collins F. S., 1909 - The green algae of North America, Tufts College Studies Scientifical, 2, 79-480.

10. Van Dam H., Mertens A. and Sinkeldam J., 1994 - A coded checklist and ecological indicator values of freshwater diatoms from the Netherlands, Netherlands Journal of Aquatic Ecology, 28, 1, 117-133, doi.org/10.1007/BF02334251.

11. Edler L. and Elbrächter M., 2010 - The Utermöhl method for quantitative phytoplankton analysis: Microscopic and molecular methods for quantitative phytoplankton analysis, Paris, UNESCO Publishing, 12-13.

12. Faridi M. A. F., Anjum A. R. and Anjum G., 1981 - Algae associated with alluvial gold of Indus at Attock, Geological Bulletin of University Peshawar, 14, 151-157.

13. Huestedt F., 1938-1939 - Systematish und Okologische Untersuchungen Uber die Diatomeenflora von Java, Bali und Sumatra, Archiv für Hydrobiologie, Suppliment, 15, 131177, 393-506, 638-790, 16, 1-155, 274-394. (in German)

14. Huestedt F., 1957 - Die Diatomeenflora des Flußsystems der Weser im Gebiet der Hansestadt Bremen, Abhandlungen herausgegeben vom Naturwissenschaftlichen Verein zu Bremen, 34, 181-440. (in German)

15. Hussain F., Anjum G. and Faridi M. A. F., 1985 - Seasonal variation of algae in a polluted pond of Yar Hussain, District Mardan, N. W. F. P., Pakistan Journal of Botany, 17, 253-256.

16. Hussain F., Masud S. S. M., Shah F., Hadi F., Zaman A. and Wazir S. M., 2009 - Some blue green algae from rice fields of Asota Sharif, District Swabi, Pakistan, Pakistan Journal of Plant Sciences, 15, 1, 45-57.

17. Hussain F., Anjum G., Akhtar P. and Durrani M. J., 2010 - Some members of class Xanthophyceae and Euglenophyceae from soils of Peshawar Valley, Khyber Pakhtunkhwa, Pakistan, Pakistan Journal of Plant Sciences, 16, 1, 37-42.

18. Hussain F., Yaseen T., Ali N. and Zaman A., 2011 - Freshwater algae of Tehsil Katlang, District Mardan, Pakistan, Pakistan Journal of Plant Sciences, 17, 2, 143-152.

19. Hussain F., Shah S. and Ahmad I., 2012 - Freshwater algae from Karnal Sher Khan Kili, District Swabi, Pakistan, Pakistan Journal of Plant Sciences, 18, 1, 47-53.

20. Khan T. M. and Faridi M. A. F., 1977 - Zygnema and Zygnemopsis in Peshawar, Pakistan Journal of Botany, 9, 1, 67-75.

21. Khuram I., Ahmad N., Jan S. and Barinova S., 2014 - Freshwater green algal biofouling of boats in the Kabul River, Pakistan, Oceanological and Hydrobiological Studies, 43, 4, 329-336. 
22. Khuram I., Barinova S., Ahmad N., Ullah A., Siraj U. D., Jan S. and Hamayun M., 2017 Ecological assessment of water quality in the Kabul River, Pakistan, using statistical methods, Oceanological and Hydrobiological Studies, 46, 2, 140-153, DOI: https://doi.org/10.1515/ohs2017-0015.

23. Novakovsky A. B., 2004 - Abilities and base principles of program module “GRAPHS”, Scientific Reports of Komi Scientific Center, Ural Division of the Russian Academy of Sciences, 27, 1-28.

24. Prescott G. W., 1962 - Algae of the Western Great Lakes area, Dusuoue: W. M. C. Brown Company, Iowa, 1004.

25. Rasiga A., Momeu L., Péterfi Ş. and Kozma A., 1999 - Biodiversity of algae of the upper and middle course of the Olt River, Transylvanian Review of Systematical and Ecological Research, The Upper and Middle Olt River Basin, 1, 31-48.

26. Sarim F. M., 1989a - Phacus in Peshawar, Peshawar University Teacher`s Association Journal, 13, 47-48.

27. Sarim F. M. 1989b - Euglena in Swat Valley, Pakistan, Peshawar University Teacher`s Association Journal, 13, 49-50.

28. Sarim F. M. 1991 - Further notes on the Charales of Pakistan, Pakistan Journal of Scientific and Industrial Research, 34, 9, 348-351.

29. Sarim F. M. and Faridi M. A. F., 1976 - Closterium in Peshawar Valley, Pakistan Journal of Botany, 8, 2, 221-239.

30. Sarim F. M. and Ayaz M., 2000 - Genes Chara from Malakand Division, N. W. F. P., Pakistan, Pakistan Journal of Plant Sciences, 6, 1, 1-7.

31. Sarim F. M. and Zaman A., 2005 - Some freshwater algae of District Charsadda, N. W. F. P., Pakistan, Peshawar University Teacher`s Association Journal, 12, 5-10.

32. Sarim F. M., Khair-un-Nisa and Afzal N., 2008 - Some freshwater algae of Tarbela Dam, Pakistan, Pakistan Journal of Plant Sciences, 14, 35-39.

33. Sarim F. M., Ali N. and Ahmad I., 2011 - Some Bacillariophyta from District Nowshera, Pakistan, Pakistan Journal of Plant Sciences, 17, 2, 137-140.

34. Stevenson J., 2014 - Ecological assessments with algae: a review and synthesis, Journal of Phycology, 50, 437-461, doi:10.1111/jpy.12.

35. Tiffany L. H. and Britton M. E., 1952 - The Algae of Illinois, Chicago: Chicago University Press, 407.

36. Transeau E. N., 1951 - The Zygnemataceae (fresh-water Conjugate Algae) with keys for the identification of genera and species: and seven hundred eighty-nine illustrations, Columbus Ohio State University Press, 321.

37. Watanabe T., Asai K. and Houki A., 1986 - Numerical estimation of organic pollution of flowing water by using the epilithic diatom assemblage - Diatom Assemblage Index (DAIpo), Science of Total Environment, 55, 209-218, doi.org/10.1016/0048-9697(86)90180-4.

38. Wehr J. D., 2002 - Freshwater algae of North America: ecology and classification, Academic Press, 917.

39. Wessa P., 2018 - Free statistics software, Office for Research Development and Education, version 1.2.1, URL https://www.wessa.net/ 